\title{
Future Medical Research Challenges
}

\author{
Hamid Yahiya Hussain* \\ Dubai Health Authority \\ *Corresponding author: Hamid Yahiya Hussain, professior, Dubai, United Arab Emirates
}

Submission: 眥 July 03, 2017; Published: 眥 August 18, 2017

\section{Opinion}

Due to tremendous scientific development in areas not included previously in traditional clinical research (like preventive medicine and biotechnology), this will generate significant opportunities to address clinical problems from new look and in new approaches. As an example, advancement in handling computational power and clearly increased use of data on human genomics opens the doors towards personalized medicine and individualized treatments designed for the individual patient, moreover, moving the concerns from treatment to prevention of disease. Likewise technological advances expecting however to create new research dilemmas by raising complex ethical questions (like, those adhering to privacy issues and genetic manipulations). Such a complexity shall definitely need to be properly addressed to avoid fragile the legitimacy of medical research and throwing it away from the advantages that advances in research can bring to society. More far, the issues related to prioritization are still an issue of complexity within the healthcare system. As it is well known that Improving health creates economic growth, yet health and economy are operating in other modality: the health of those with high socioeconomic status improve better than individuals with lower socio-economic status. Which is reflected as health inequality and emerging as an important socio-economic challenge in both developed and developing countries, since health interests are is not equally and adequately distributed across the whole population in the society. As one of the future major concerns regarding pure medical science and researches, is that the main 'market' for pure medical science would be the applied medical scientists, who need radical strategies to solve problems which are not yielding to established methods. And achieve that goal, to create elite pure medical science bodies, that might come from the leadership of academic 'entrepreneurs' (for instance, imaginative patrons in the major funding foundations), or be triggered by a widespread public recognition of the probable exhaustion of existing applied medical science approaches to solving major therapeutic challenges. 